\title{
A Single Molecule Investigation of I-Motif: Stability, Folding Kinetics, and Potential as an In-situ pH Sensor
}

Golam Mustafa, Prabesh Gyawali, Jacob A. Taylor, Parastoo Maleki, Marlon V. Nunez, Michael

\author{
C. Guntrum, Hamza Balci* \\ Department of Physics, Kent State University, Kent, OH 44242 \\ * To whom correspondence should be addressed: Tel: +1-330-672-2577; \\ Email: hbalci@kent.edu
}

\begin{abstract}
We present a collection of single molecule work on the i-motif structure formed by the human telomeric sequence. Even though it was largely ignored in earlier years of its discovery due to its modest stability and requirement for physiologically low $\mathrm{pH}$ levels $(\mathrm{pH}<6.5)$, the i-motif has been attracting more attention recently as both a physiologically relevant structure and as a potent $\mathrm{pH}$ sensor. In this manuscript, we establish single molecule Förster resonance energy transfer (smFRET) as a tool to study the i-motif over a broad $\mathrm{pH}$ and ionic conditions. We demonstrate $\mathrm{pH}$ and salt dependence of i-motif formation under steady state conditions and illustrate the kinetics of i-motif folding in real time at the single molecule level. We also show the prominence of intermediate folding states and reversible folding/unfolding transitions. We present an example of using the i-motif as an in-situ $\mathrm{pH}$ sensor and use this sensor establish the time scale for the $\mathrm{pH}$ drop in a commonly used oxygen scavenging system.
\end{abstract}

\section{Introduction}

Depending on the nucleic acid sequences and environmental conditions, DNA can form secondary structures other than the canonical double helix structure. These non-canonical structures include G-quadruplex (GQ) and intercalated motif (i-motif) which form depending on temperature, type and concentration of cations present, and $\mathrm{pH}^{1-8}$. The cytosine rich (C-rich) sequences can adopt i-motif structure that is formed by two parallel duplexes which are held together by intercalated cytosine-hemiprotonated cytosine $\left(\mathrm{C}: \mathrm{CH}^{+}\right)$base pairs ${ }^{1,9-11}$. The structure also has three loop regions that are unstructured (Figure 1A).

Biophysical studies revealed that the stability of the i-motif depends on the sequence ${ }^{7}$ and length of the loops where i-motif with shorter loops are more stable ${ }^{12}$. The stability also depends on the environmental condition such as $\mathrm{pH}^{13}$, temperature ${ }^{8}$, ionic strength ${ }^{8,14,15}$, cationic effects 
15-17 and molecular crowding ${ }^{18,19}$. The structure of i-motif DNA in solution at various $\mathrm{pH}$ conditions was investigated by using synchrotron small-angle X-ray scattering technique ${ }^{13}$.

As protonation is required for $\mathrm{C}: \mathrm{CH}^{+}$base pair formation, the i-motif structure shows a sharp transition from folded i-motif to random coil (unfolded) conformation around $\mathrm{pH} 6.5$, below which it remains folded ${ }^{9-11,20,21}$. Formation, stability, and kinetics of i-motif structures at physiological $\mathrm{pH}$ are not only of fundamental interest, but also potentially significant for sensor applications. Several studies have now established that it is possible to stabilize i-motif structures at physiological $\mathrm{pH}$ under molecular crowding conditions ${ }^{18,22,23}$, in a negative supercoiling DNA template ${ }^{24}$, in the presence of silver(I) cations ${ }^{25}$, or in the presence of carboxyl-modified single walled carbon nanotubes ${ }^{26}$. The reversible and fast transformability of the C-rich sequences with $\mathrm{pH}$, from unfolded to a folded i-motif, has potential applications in designing DNA nanomachines 10,27,28, logic operation switches ${ }^{29-31}$, electrochemical sensors for proton detection ${ }^{32}$ and cellular $\mathrm{pH}$ sensors ${ }^{33,34}$. By introducing structural changes within the DNA constructs, it is possible to tune the $\mathrm{pH}$ range that can be sensed by the i-motif and make it a more versatile $\mathrm{pH}$ sensor.

Moreover, the i-motif structure has been observed in the nuclei of human cells ${ }^{35}$, which gives significant support for the physiological relevance of these structures. C-rich sequences that can fold into i-motif are found in telomeres ${ }^{36}$, in centromeres ${ }^{37}$, and near the promoter region of oncogenes ${ }^{38-40}$. Formation of i-motif has been shown to influence DNA replication ${ }^{41}$ and transcription ${ }^{42-45}$. For an extensive description of the roles of i-motif structures in the cell, we refer the reader to an excellent recent review ${ }^{46}$.

Folding and stability of the i-motif have been studied as a function of $\mathrm{pH}$ and ionic conditions using bulk biophysical methods ${ }^{8,9,46}$. However, limited work has been performed using single molecule methods ${ }^{47-50}$. In this study, we used single molecule Förster resonance energy transfer (smFRET) to investigate stability, kinetics, intermediate folding states, and evolution of imotif formation at different $\mathrm{pH}$ and ionic conditions. After introducing the construct and the method in Figure 1, we demonstrate the capabilities of our approach in Figure 2 and Figure 3 clearly identifying the i-motif structure and evolution of its folding over a broad $\mathrm{pH}$ and salt concentration range. In Figure 4, we took advantage of our single molecule approach to monitor the folding dynamics of i-motif in real time using $\mathrm{pH}$ jump experiments. These measurements clearly demonstrate the presence of at least one prominent intermediate folding state that is populated during most of folding events. In Figure 5 and Figure 6, we present proof-of-principle 
measurements that demonstrate the capability of the i-motif structure as an in-situ $\mathrm{pH}$ sensor for smFRET studies. We demonstrate that the i-motif structure can sense the time-dependent drop in $\mathrm{pH}$ of the environment when "gloxy" (glucose oxidase plus catalase), the most commonly used oxygen scavenging system for single molecule fluorescence studies, is used to enhance photostability of the dyes.

\section{Materials and Methods}

\section{DNA Constructs.}

The following modified (fluorescently labeled and biotin conjugated) DNA oligonucleotides were purchased as HPLC purified from Integrated DNA Technologies (Coralville, IA, USA):

Stem Strand: / Biotin / GCCTCGCTGCCGTCGCCA-Cy5

i-motif Strand: Cy3-TTCCCTAACCCTAACCCTAACCCTTTGGCGACGGCAGCGAGGC

The underlined nucleotides form the i-motif structure. A partial duplex DNA (pdDNA) construct that has an 18-bp duplex stem and a single-stranded overhang, which forms the i-motif, was prepared by annealing the stem strand and the i-motif strand at $90{ }^{0} \mathrm{C}$ for 3 min followed by cooling to room temperature over 3 hours. The annealing was performed in $150 \mathrm{mM} \mathrm{KCl}$ and $10 \mathrm{mM} 2-$ ethanesulfonic acid, also known as MES, at pH 7.0.

\section{Sample preparation and smFRET assay}

Laser-drilled quartz slides and glass coverslips were cleaned, including a piranha etching step, and coated with polyethylene-glycol (PEG) to prevent non-specific binding of DNA molecules to the surface. A mixture containing a ratio of 100:2 of m-PEG-5000: biotin-PEG-5000 (purchased from Laysan Bio Inc.) was used to coat the surface. The sample chamber was then prepared by sandwiching a double-sided tape between these PEGylated slides and coverslip and treated with $0.01 \mathrm{mg} / \mathrm{ml}$ neutravidin, which enabled immobilization of biotin-tagged pdDNA constructs on the biotin-PEG surface. This surface cleaning and DNA immobilization steps are similar to those reported previously ${ }^{51}$.

A solution of $50 \mathrm{pM}$ pdDNA was diluted from the $1 \mu \mathrm{M}$ annealing stock in multiple steps and was incubated in the sample chamber for 3-5 min at $150 \mathrm{mM} \mathrm{KCl}$ and $10 \mathrm{mM}$ MES (pH 7.0). The chamber was then washed with $10 \mathrm{mM}$ MES ( $\mathrm{pH}$ 7.0) to remove excess pdDNA. Approximately 250-280 molecules/imaging area $\left(\sim 4 \times 10^{3} \mu \mathrm{m}^{2}\right)$ were obtained with this procedure. For all measurements other than the $\mathrm{pH}$ jump experiments presented in Figure 4, 20 
short movies of 15 frames/movie were recorded at frame acquisition rate of $100 \mathrm{~ms} /$ frame for each assay condition. Longer movies (2000-4000 frames at $31 \mathrm{~ms} /$ frame) were recorded for $\mathrm{pH}$ jump experiments where folding dynamics was studied.

We used two different imaging solutions for our measurements. For all the data, except those presented in Figure 4, the imaging solution contained $50 \mathrm{mM}$ MES of indicated pH, $2 \mathrm{mM}$ Trolox, $25 \mathrm{mM}$ protocatechuic acid (PCA), $0.35 \mathrm{mg} / \mathrm{ml}$ protocatechuate-3,4-dioxygenase (PCD), $0.1 \mathrm{mg} / \mathrm{ml}$ bovine serum albumin (BSA), $2 \mathrm{mM} \mathrm{MgCl}$, and indicated concentrations of $\mathrm{KCl}$. This solution will be referred to as PCA/PCD imaging buffer. When preparing the buffers, we ensured the $\mathrm{pH}$ is stable to three decimal points, but for conciseness of the reporting, we rounded these to the nearest one decimal point, e.g. pH 6.324 was reported as 6.3. The only exception to this is when equilibrium constants were reported. For these, we reported two decimal points, e.g. 6.32, to demonstrate the variation between different conditions within the uncertainties of the measurement $( \pm 0.05$ or less in $\mathrm{pH})$. Trolox increases brightness of the fluorophores by quenching their dark triplet state. PCA and PCD form an oxygen scavenging system that delays photobleaching of fluorophores. BSA patches the surface areas that might have imperfect PEGylation. To allow the system to reach steady state and PCA/PCD to reduce the oxygen concentration to adequate levels, the imaging solution was incubated with the DNA molecules for $30 \mathrm{~min}$ before measurement.

For the $\mathrm{pH}$ jump measurements presented in Figure 4 glucose oxidase and catalase (also called gloxy) was used as the oxygen scavenging system instead of the PCA/PCD system. The contents of the imaging buffer were otherwise same as listed above. This solution will be referred to as gloxy imaging buffer. A significant advantage of gloxy, compared to PCA/PCD, is that long incubation times are not required to remove oxygen, and data acquisition can effectively start as soon as the imaging buffer is introduced into the chamber. This enables observing folding of the i-motif in real time as movies could be recorded while $\mathrm{pH}$ of the environment was dropped from 7.0 to 5.3 via a buffer exchange (called pH jump in this study and "flow measurement" in some others where structural changes in DNA were recorded in real time ${ }^{52}$ ). For these measurements, the DNA molecules were first incubated in a PCA/PCD imaging buffer that contains $50 \mathrm{mM}$ MES at $\mathrm{pH} 7.0$ and $150 \mathrm{mM} \mathrm{KCl}$. After $30 \mathrm{~min}$ of incubation, recording of a long movie of 2000-4000 frames at an acquisition rate of $31 \mathrm{~ms} /$ frame was initiated. After recording 200 frames, a gloxy imaging buffer containing $50 \mathrm{mM}$ MES at pH 5.3 (all other ingredients were kept identical) was flown into the sample chamber, while the recording continued. 
Even though gloxy made the $\mathrm{pH}$ jump experiments possible, it is known to result in a gradual $\mathrm{pH}$ drop in the chamber, due to generation of gluconic acid as the by-product of reducing the free oxygen concentration according to the following reaction:

$$
\begin{gathered}
\beta \text {-D-glucose }+\mathrm{O}_{2}+\mathrm{H}_{2} \mathrm{O} \stackrel{\text { glucose oxidase }}{\longrightarrow} \text { gluconic acid }+\mathrm{H}_{2} \mathrm{O}_{2} \\
\mathrm{H}_{2} \mathrm{O}_{2} \stackrel{\text { catalase }}{\longrightarrow} \mathrm{H}_{2} \mathrm{O}+\frac{1}{2} \mathrm{O}_{2}
\end{gathered}
$$

The rate of the $\mathrm{pH}$ drop depends on the buffer strength, which we quantified in Figure 5 by using the i-motif as an in-situ $\mathrm{pH}$ sensor. These measurements started from physiological $\mathrm{pH}$ (7.5) which motivated the use of Tris- $\mathrm{HCl}$ as the buffer instead of MES due to its higher $\mathrm{pKa}^{53}$. The imaging solution contained the following for these measurements: Indicated concentration of Tris- $\mathrm{HCl}$ (initial $\mathrm{pH} 7.5$ ), $0.1 \mathrm{mg} / \mathrm{ml}$ glucose oxidase, $0.02 \mathrm{mg} / \mathrm{ml}$ catalase, $2 \mathrm{mM}$ Trolox, $0.1 \mathrm{mg} / \mathrm{ml} \mathrm{BSA}$, $2 \mathrm{mM} \mathrm{MgCl}_{2}$, and $150 \mathrm{mM} \mathrm{KCl}$.

\section{Imaging Setup}

A prism-type total internal reflection fluorescence microscope equipped with an Olympus IX-71 microscope and an Andor IXON EMCCD camera (IXON DV-887 EMCCD, Andor Technology, CT, USA-now part of Oxford Instruments) was used for the measurements. The donor fluorophore was excited with $532 \mathrm{~nm}$ wavelength green laser (Spectra Physics Excelsior). An Olympus water objective (60x, 1.20 NA) was used for collecting the fluorescence signal.

\section{Data Analysis}

Using a custom software written in $\mathrm{C}++$, the recorded movies were analyzed and time traces of donor intensity $\left(\mathrm{I}_{\mathrm{D}}\right)$ and acceptor intensity $\left(\mathrm{I}_{\mathrm{A}}\right)$ for each molecule were generated. The FRET efficiency (EFRET) was calculated using $E_{F R E T}=I_{A} /\left(I_{A}+I_{D}\right)$. The FRET efficiency population histograms were constructed from single molecule traces such that each molecule contributed equally, regardless of how long the molecules remained fluorescent (time it took for molecules to photobleach). The leakage of the donor signal into the acceptor channel, which results in the donor-only (DO) peak, was subtracted from the histograms and the DO EFRET level was shifted to zero to correct for this leakage.

In order to analyze the $\mathrm{pH}$ and salt concentration dependence of the i-motif structure, we utilized a published method ${ }^{7}$. Accordingly, an equilibrium constant $K$ is defined as:

$$
K=\frac{\text { folded population }}{\text { unfolded population }}=\frac{\text { fraction } i \text {-motif }}{1-(\text { fraction } i \text {-motif })}
$$


When the folded and unfolded populations are equal $K=1$. The $\mathrm{pH}$ or salt concentration where $K=1$ is satisfied will be referred to as $K_{e q}$. To determine $K_{e q}$, we plot $\log (K)$ vs. pH or $\log (K)$ vs. $\log$ (salt concentration), and linearly fit the most rapidly varying segments of the curve. When $K=1 \Rightarrow \log (K)=0$ so $\mathrm{pH}$ or salt concentration where the line crosses 0 will be the corresponding $\mathrm{K}_{\mathrm{eq}}$. The slope of $\log (K)$ vs. $\mathrm{pH}$ has been interpreted as the number of protons gained or lost during the folding or unfolding transitions, respectively ${ }^{7}$.

Origin software program was used for these analyses. The errors in the cited $K_{e q}$ values are the standard deviations obtained from the fitting analysis.

A

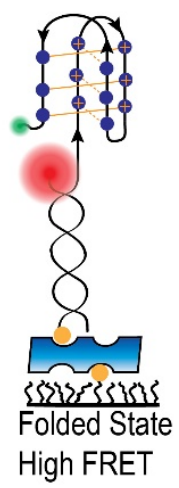

- Cy 5 - Biotin PEG - C

- $\mathrm{Cy} 3$ Neutravidin $\mathrm{CH}^{+}$

C

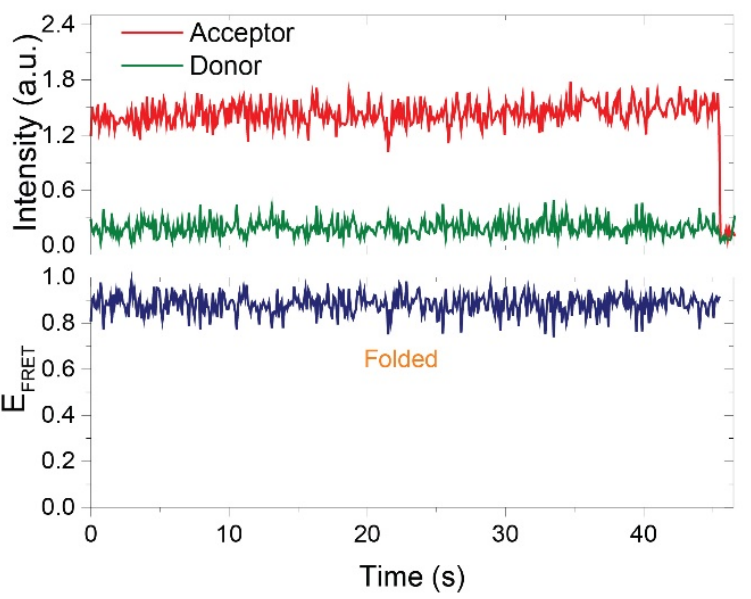

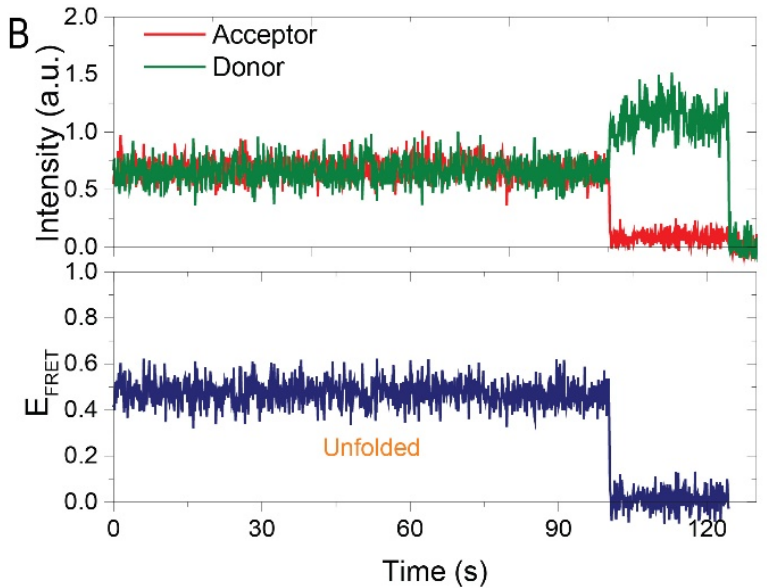

D

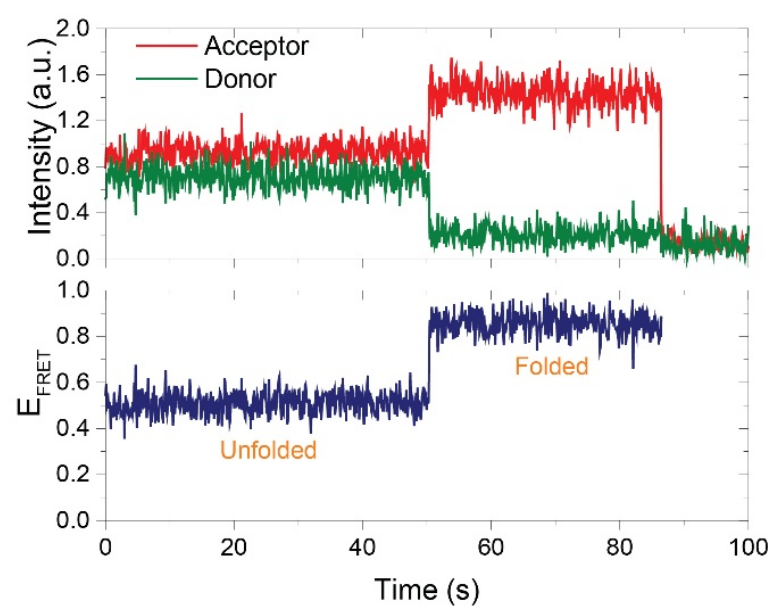

Figure 1 A schematic of the DNA construct and an example smFRET time trace. (A) A partial duplex DNA construct that has an acceptor (Cy5) on the short strand and a donor (Cy3) at the free-end of the long strand. Folding of the DNA into i-motif brings the donor-acceptor fluorophores closer to each other and results in an increase in the FRET efficiency. (B)-(D): Example SmFRET traces that show unfolded state $(B)$, folded state $(C)$, and a transition from the unfolded state to folded state $(D)$. 


\section{Results and Discussion}

\section{I-motif stability as a function of pH}

In this section, we present smFRET studies that demonstrate the capabilities of the method to distinguish between the folded and unfolded i-motif structures under different $\mathrm{pH}$ and ionic conditions. Figure 1A shows a schematic of the DNA construct and the donor-acceptor dye positions. Figure 1B-D show example smFRET time traces that show unfolded state at FRET efficiency $\mathrm{EFRET}_{\mathrm{f}}=0.5$ (Figure 1B), folded state at EFRET $=0.9$ (Figure 1C) and a transition from the unfolded to the folded state where the FRET efficiency rises from EFRET $\approx 0.5$ to EFRET $\approx 0.9$ (Figure 1D). Histograms of time traces, each containing data from several thousand molecules, show two well-separated peaks representing the folded and unfolded states (Figure 2).

Figure 2 shows $\mathrm{pH}$ titration (pH 5.9 to 7.0) at $20 \mathrm{mM}$ (Figure 2A-B), $150 \mathrm{mM}$ (Figure 2CD), and $300 \mathrm{mM} \mathrm{KCl} \mathrm{(Figure} \mathrm{2E-F).} \mathrm{Five} \mathrm{smFRET} \mathrm{histograms} \mathrm{are} \mathrm{shown} \mathrm{for} \mathrm{each} \mathrm{KCl}$ concentration ([KCl]). In these histograms, the high FRET states (EFRET $>0.70)$ represent the folded i-motif and low FRET states $\left(\mathrm{EFRET}_{\mathrm{F}}<0.60\right)$ represent the unfolded state. As $\mathrm{pH}$ is lowered the folded state population increases while the unfolded state population decreases, as expected. For all tested salt conditions, all molecules were folded at pH 5.9 (a single peak at EFRET $\approx 0.80$ ) while they were all unfolded at pH 7.0 (a single peak at $\mathrm{E}_{\mathrm{FRET}} \approx 0.50$ ). Between $\mathrm{pH} 6.0$ to 6.6, the entire population transitions from i-motif to random coil, in agreement with earlier reports ${ }^{9}$. The steady state variation of folded state as a function of $\mathrm{pH}$ is illustrated in Figure $2 \mathrm{~B}$ for $20 \mathrm{mM} \mathrm{KCl}$, in Figure 2D for $150 \mathrm{mM} \mathrm{KCl}$, and in Figure $2 \mathrm{~F}$ for $300 \mathrm{mM} \mathrm{KCl}$. The $\mathrm{pH}$ equilibrium constants $\left(\mathrm{K}_{\mathrm{eq}}\right)$, where $50 \%$ of molecules are folded, were determined by linear fits (blue lines in the inset of cited figures) to $\log (K)$ vs. $\mathrm{pH}$ data. These fits resulted in the following: $\mathrm{K}_{\mathrm{eq}}=6.38 \pm 0.05, \mathrm{~K}_{\mathrm{eq}}=6.23 \pm 0.05$ and $\mathrm{K}_{\mathrm{eq}}=6.17 \pm 0.05$ for $20 \mathrm{mM}, 150 \mathrm{mM}$, and $300 \mathrm{mM} \mathrm{KCl}$, respectively. Higher concentrations of $\mathrm{K}^{+}$require lower $\mathrm{pH}$ values for $\mathrm{i}$-motif formation, as expected by Le Chatelier's principle ${ }^{8}$. The uncertainties were based on the $\mathrm{pH}$ range that results in $\pm 5 \%$ variation in the fraction of $\mathrm{i}$-motif population. The equilibrium constants we observe (for example $\mathrm{K}_{\mathrm{eq}}=6.23 \pm 0.05$ at $150 \mathrm{mM} \mathrm{KCl}$ ) are slightly higher than those observed in bulk measurements. For example, circular dichroism measurements reported $\mathrm{K}_{\mathrm{eq}} \approx 5.75$ and $\mathrm{K}_{\mathrm{eq}} \approx 5.85$ at $165 \mathrm{mM}$ and $115 \mathrm{mM} \mathrm{KCl}$, respectively, which can be interpolated to $\mathrm{K}_{\mathrm{eq}} \approx 5.8$ at $150 \mathrm{mM} \mathrm{KCl}^{8}$. The higher $\mathrm{K}_{\text {eq }}$ in our measurements, where DNA molecules are immobilized on the surface, might be due to surface-induced stabilization of the i-motif observed for some surfaces ${ }^{54}$. 

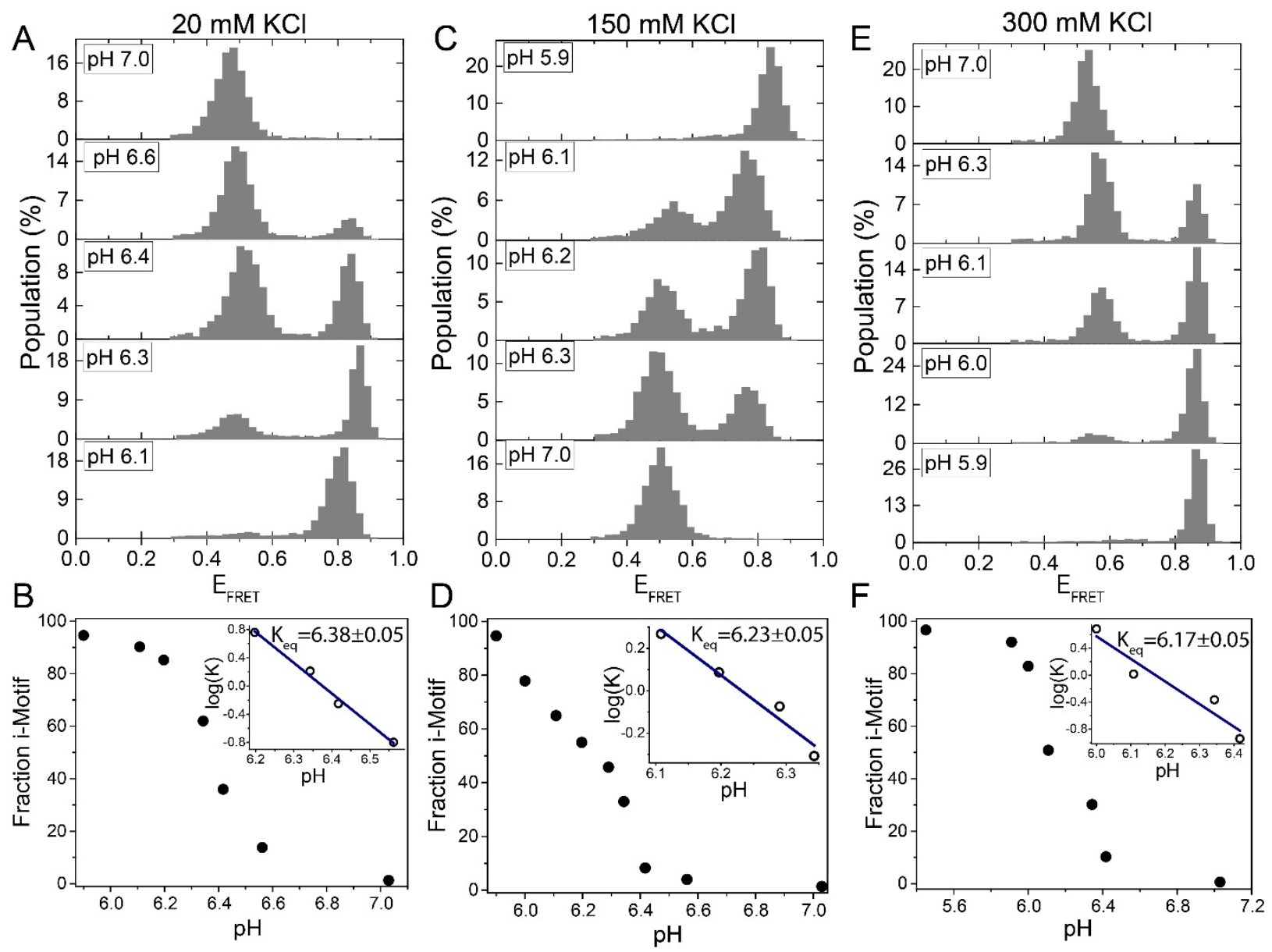

Figure 2 Titration of $\mathrm{pH}$ at a constant $\mathrm{KCl}$ concentration. The pH was titrated from 7.0 to 5.9 for (A)-(B) $20 \mathrm{mM} \mathrm{KCl}$; (C)-(D) 150

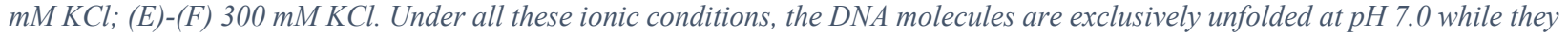
are exclusively folded at $\mathrm{pH}$ 5.9. At intermediate $\mathrm{pH}$ values around $\mathrm{pH}$ 6.3, we observe two peaks that represent the folded or unfolded states. (B), (D), and (F) show the evolution of the fraction of $i$-motif molecules as a function of $p H$. Blue lines in the insets are linear fits to the most rapidly varying segments of the curve of $\log (K)$ vs. $p H$ data.

\section{I-motif stability as a function of ion concentration}

We also studied the fraction of folded i-motif structures as a function of $[\mathrm{KCl}]$ as shown in Figure 3A-B. The $\mathrm{pH}$ was fixed at 6.3 for these measurements while $[\mathrm{KCl}]$ was titrated from 0 $1000 \mathrm{mM}$. As expected, the unfolded state population increases as $[\mathrm{KCl}]$ is increased. It was possible to drive the system from almost completely folded to almost completely unfolded between 80-400 $\mathrm{mM} \mathrm{KCl}$, in agreement with earlier studies 9 . As Figure 3A shows, the peak that represents the unfolded state gradually shifts to higher FRET values as $[\mathrm{KCl}]$ is increased, which is expected due to better shielding of the negatively charged ssDNA backbone at these higher salt concentrations. This results in the folded and unfolded peaks to approach each other since the shift in the folded state is significantly less prominent, which is also expected since unstructured ssDNA 
forms only a small fraction of the overall construct (two spacer nucleotides on either side of the imotif). This could potentially prevent smFRET to be as sensitive to i-motif formation at very high salt concentrations (several molar); however, this is not the case until $1 \mathrm{M} \mathrm{KCl}$. Several molar salt concentrations would require very low $\mathrm{pH}$ values for i-motif formation, where photostability of fluorophores commonly used in smFRET measurements is typically poor in our experience. Therefore, it would be challenging to study i-motif structure and kinetics using smFRET at such high salt concentrations. Figure 3B shows the evolution of fraction of i-motif population as function of $\mathrm{KCl}$. A linear fit to $\log (K)$ vs. $\log ([K C l])$ data, shown in the inset of Figure $3 \mathrm{~B}$, resulted in $\mathrm{K}_{\mathrm{eq}}=215 \pm 3 \mathrm{mM} \mathrm{KCl}$ at $\mathrm{pH}$ 6.3. Since the $\mathrm{i}$-motif is closely related to GQ, and the GQ stability shows a strong dependence on the ion type $\mathrm{e}^{55}$, we performed similar salt titration measurements using $\mathrm{NaCl}$ (Figure 3C-D). Unlike the GQ, our analysis demonstrated very similar i-motif stabilities for $\mathrm{K}^{+}$or $\mathrm{Na}^{+}$, with $\mathrm{K}_{\mathrm{eq}}=198 \pm 3$ for $\mathrm{NaCl}$.

\section{Monitoring i-motif folding in real time}

In order to observe the folding of the i-motif in real time, we performed buffer exchange measurements where a lower $\mathrm{pH}$ buffer $(\mathrm{pH} 5.3)$ was injected into the sample chamber that contained unfolded molecules at a higher $\mathrm{pH}(\mathrm{pH}$ 7.0). Gloxy, rather than PCA/PCD, which was used for the data in Figure 2 and Figure 3, was used in these measurements as the oxygen scavenging system in order to image the folding process in real time, which happens within a few seconds of the buffer exchange. The lower $\mathrm{pH}$ buffer was injected into the chamber with a syringe pump that minimized the disturbance on the system and allowed continuous imaging of the folding process. Figure 4A shows a representative smFRET time trace that captures the folding process. The trace shows an intermediate folding state, which was detected by Hidden-Markov modeling (magenta line that are overlaid on the data), performed using the vbFRET program ${ }^{56}$. Figure 4B shows the transition density plot (TDP) constructed from 105 such traces. In TDP, the x-axis represents the FRET state before the transition, initial FRET, while the y-axis represents the FRET state after the transition, final FRET. The color in TDP represents the number of transitions, red being the most frequent and blue the least. For example, a folding transition from $\mathrm{E}_{\mathrm{FRET}} \approx 0.40$ to $\mathrm{EFRET}_{\mathrm{F}} \approx 0.70$, it will contribute as one unit in TDP at point $(0.40,0.70)$. Similarly, an unfolding transition from $\mathrm{E}_{\mathrm{FRET}} \approx 0.70$ to $\mathrm{E}_{\mathrm{FRET}} \approx 0.40$ will contribute to the contour plot at point $(0.70,0.40)$. The white dashed line indicates the $45^{\circ}$. Transitions above this line are due to folding while transitions below it are due to unfolding. As expected, the levels above the $45^{\circ}$-line are more 
populated as a higher $\mathrm{pH}$ buffer was exchanged with a lower $\mathrm{pH}$ buffer and the transitions were biased in the folding direction. However, it should be noted that there are clearly detectable states below the $45^{\circ}$-line indicating that the folding process is not irreversible, and some molecules occasionally unfold during this transition period.
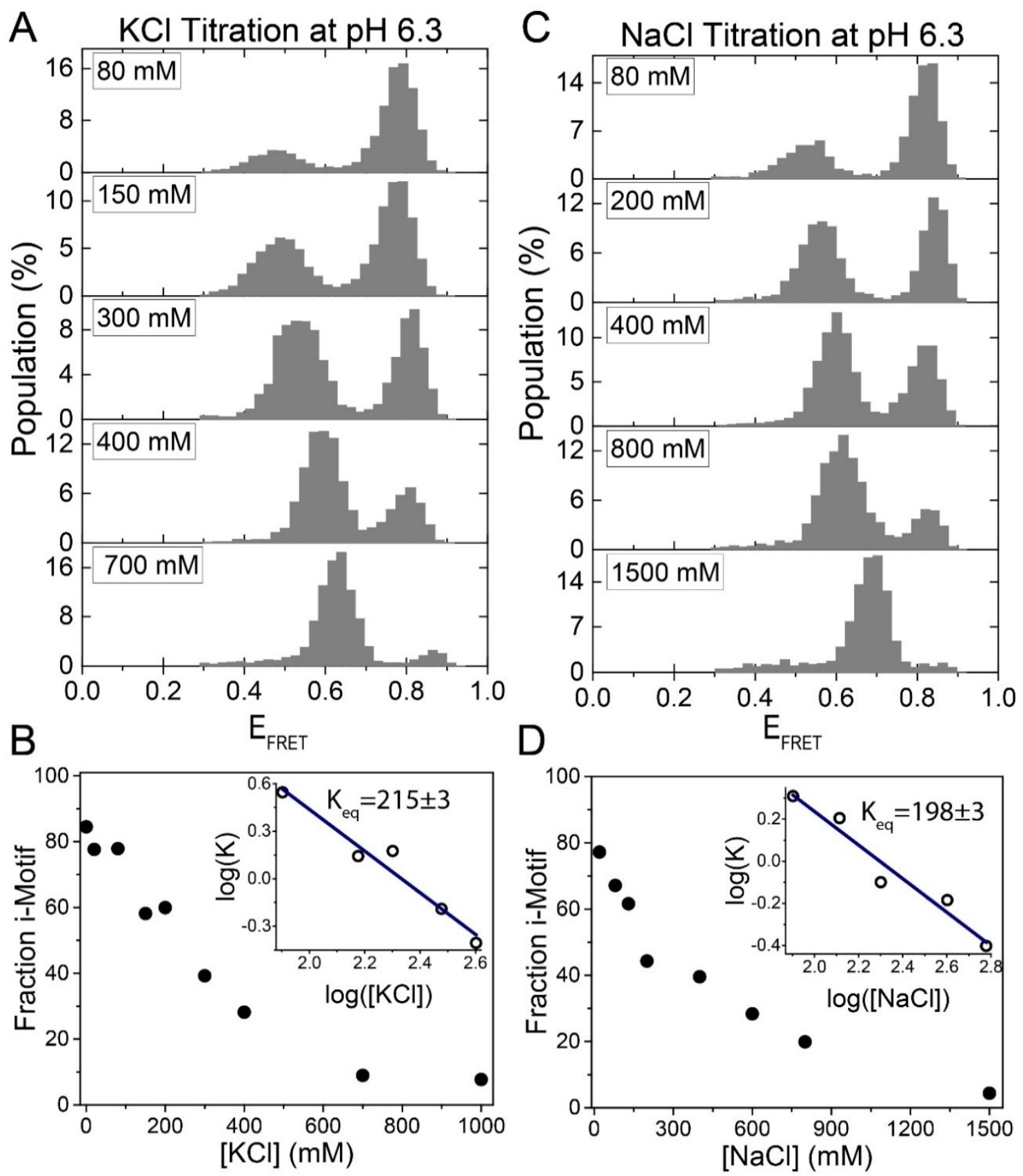

Figure 3 Titration of $\mathrm{KCl}$ or $\mathrm{NaCl}$ at $\mathrm{pH}$ 6.3. $\mathrm{KCl}$ was titrated between 0-1000 mM shown in (A) and (B) while NaCl titration is shown in $(C)$ and $(D)$. While most molecules are folded at low salt concentration, they gradually unfold as the salt concentration is increased. (B) and (D) show fraction of $i$-motif molecules as a function of [KCl] or [NaCl], respectively. Blue lines in the insets are linear fits to the most rapidly varying segments of the curve of $\log (K) v s$. $\log$ (salt concentration) data. 
Another interesting revelation of the TDP is the presence of prominent intermediate states, which was not observed in steady state histograms. These distinct transitions are marked with white dashed circles, which are numbered as 1,2,3, and 4 in the folding direction. The reverse of these transitions in the unfolding direction are marked with 1', 2', 3', and 4', respectively. The most populated transitions in TDP are from EFRET $\approx 0.40$ to EFRET $\approx 0.55$ (circle 1 ). The molecules that reach EFRET $\approx 0.55$ either continue to fold and reach EFRET $\approx 0.70$ (Circle 3 ) or EFRET $\approx 0.75$ (Circle 4). There also molecules that directly transition from the unfolded state EFRET $\approx 0.40$ to $\mathrm{EFRET}_{\mathrm{F}} \approx 0.70$ (Circle 2). All of these transitions are reversible, in the form of transitions shown with circles 1', 2', 3', and 4'. Assuming the EFRET $\approx 0.75$ state to be the completely folded state and the $\mathrm{EFRET}_{\mathrm{F}} \approx 0.40$ state the completely unfolded state, we do not observe any direct folding or unfolding events, i.e. we do not have transitions around $(0.40,0.75)$ or $(0.75,0.40)$. Instead, during both folding and unfolding, the molecules transition to the intermediate state first before they completely fold or unfold. As the intermediate state is a relatively short-lived state that is observed only during the transitions, it is not surprising that it was not detected in steady state histograms depicted in Figures 2 and 3, which survey the molecules after they are incubated in respective buffers for about 30 minutes. In order to amplify the contribution of the intermediate populations in the histograms, we constructed a new histogram from the time traces of these $\mathrm{pH}$ jump measurements and included only the segments spanning $2 \mathrm{~s}$ before and $2 \mathrm{~s}$ after the transition. Figure $4 \mathrm{C}$ shows the new histogram where the states between the unfolded (EFRET $\approx 0.40)$ and folded (EFRET $\approx 0.75)$ states are significantly populated (gray histogram). For reference, we overlaid the corresponding steady state histograms at pH 5.9 (red histogram) and at pH 7.0 (blue histogram). The population in the gray histogram between the blue and red peaks represents the intermediate folding state visited during the $\mathrm{pH}$ jump experiments.

\section{I-motif as an in-situ pH sensor}

The oxygen scavenging system is a critical component of single molecule fluorescence studies that use small organic fluorophores since free oxygen radicals are the primary cause of photobleaching, a chemical transition of the fluorophores to a non-fluorescent state. PCA/PCD and gloxy (glucose oxidase + catalase) are the two most commonly used oxygen scavenging systems and they have different characteristics that make them better choices for different applications, as illustrated in this study. While PCA/PCD provides a constant $\mathrm{pH}$ over multiple 
hours of imaging, it is typically necessary to incubate PCA/PCD for 10-30 minutes in the sample chamber before it becomes effective. On the other hand, gloxy reduces the free oxygen concentration much more rapidly, a few seconds, but it results in accumulation of gluconic acid which gradually reduces the $\mathrm{pH}$. Because of these characteristics, most measurements in this study were performed with PCA/PCD as having a stable $\mathrm{pH}$ was essential to avoid having a compounding factor when salt or $\mathrm{pH}$ of the environment was changed.

A

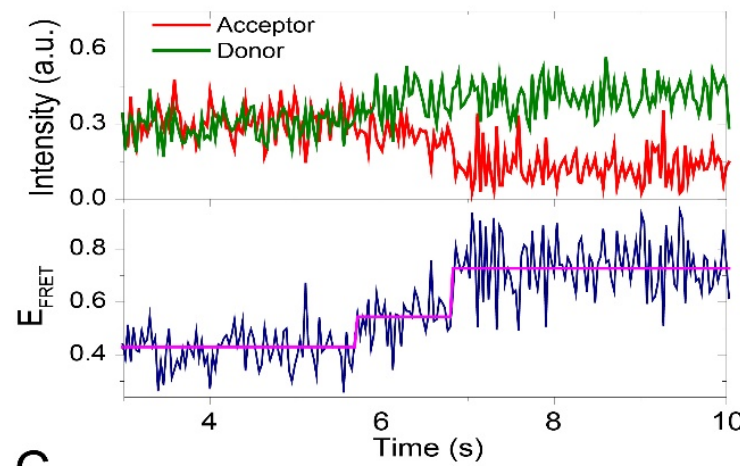

C

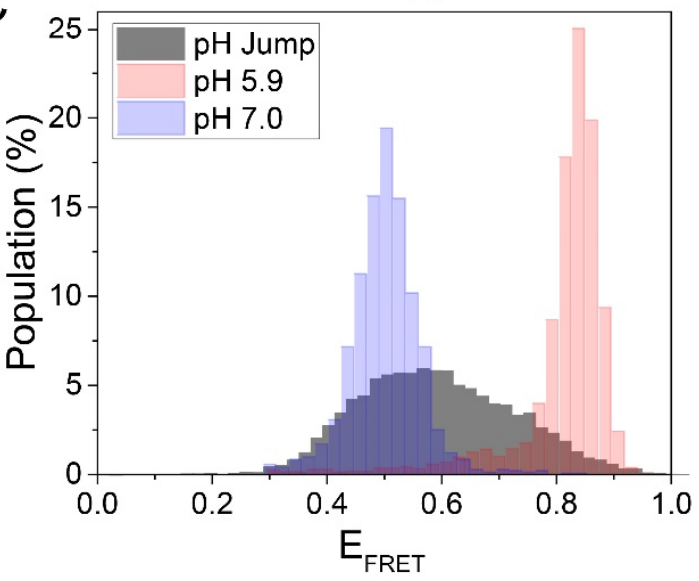

B

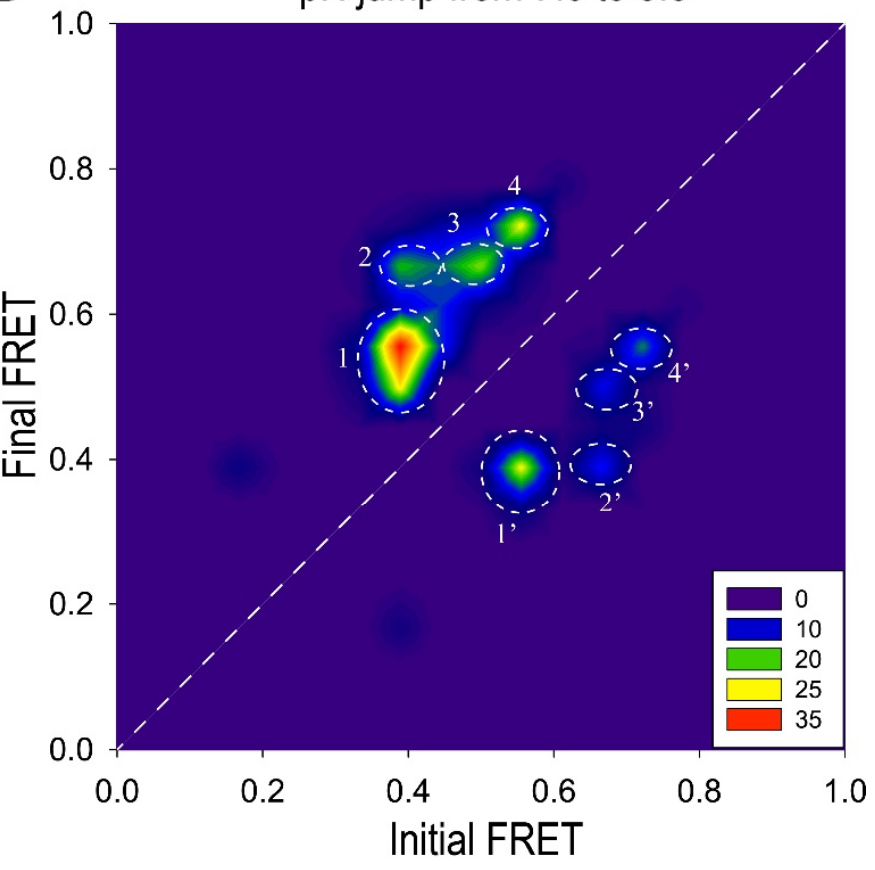

Figure 4 Real time monitoring of the folding process using $p H$ jump measurements. (A) An example time trace is shown. The DNA molecules transition from an unfolded to a folded state as the $\mathrm{pH}$ is lowered from 7.0 to 5.3. The trace shows a clear intermediate state that was detected with HMM analysis (the magenta line overlaid on the data). (B) The TDP shows a contour plot of transitions from 104 molecules. The prominent transitions are marked with white circles and numbered as 1,2,3, and 4 in the folding direction. The reverse of these transitions in the unfolding direction are numbered as 1', 2', 3', and 4'. All folding and unfolding processes go through intermediate states between the completely unfolded EFRET $\approx 0.40$ and completely folded EFRET $\approx 0.75$ states. (C) A histogram (gray) constructed from the FRET levels spanning the interval $2 \mathrm{~s}$ before and $2 \mathrm{~s}$ after the transition. This representation amplifies the contribution of intermediate states which are not present in steady state histograms obtained 30-min after the buffer exchange is made. For reference, the steady state distributions at pH 7.0 (completely unfolded) and pH 5.9 (completely folded) are shown with blue and red histograms, respectively.

In these measurements, incubating PCA/PCD in the chamber for 30 minutes was possible as we were primarily interested in the steady state distributions. However, in the $\mathrm{pH}$ jump measurements where the folding process was much faster and we were interested in the dynamics 
of this fast process, gloxy was preferred as the oxygen scavenging system. Even though fluorophore brightness and lifetime were demonstrated to be superior in PCA/PCD compared to gloxy for some of the commonly used fluorophores ${ }^{57}$, gloxy is still probably the most widely used oxygen scavenging system in single molecule fluorescence measurements due to this fast action capability. Therefore, it is important to understand the extent of the $\mathrm{pH}$ drop in gloxy as a function of time to establish time limits for justifying the assumption of relatively constant $\mathrm{pH}$ in the environment.

This important question was previously investigated by using SNARF-1, a ratiometric dual emission dye ${ }^{58}$. By characterizing the relative populations of the two peaks at different $\mathrm{pH}$ values, the $\mathrm{pH}$ variation within the sample chamber was determined. In this study, the sample chamber was placed in the path of the light beam within the spectrofluorometer and the $\mathrm{pH}$ was determined based on bulk FRET signal. In this study, we monitored the $\mathrm{pH}$ variation based on folding of the i-motif as function of time. As the $\mathrm{pH}$ drop would depend on the strength of the buffer, we performed the same measurement in $5 \mathrm{mM}, 10 \mathrm{mM}, 15 \mathrm{mM}$ and $50 \mathrm{mM}$ Tris that had an initial $\mathrm{pH}$ of 7.5. We would expect the $\mathrm{pH}$ drop to be faster in weaker buffers therefore we would expect the initially unfolded DNA strands to fold into i-motif after shorter incubation periods compared to that in stronger buffers. Based on the ionic conditions of this assay $(150 \mathrm{mM} \mathrm{KCl})$ and the calibration data presented in Figure 2C-D, we conclude that the $\mathrm{pH}$ of the environment should be 5.9 for all the DNA molecules to fold into i-motif.
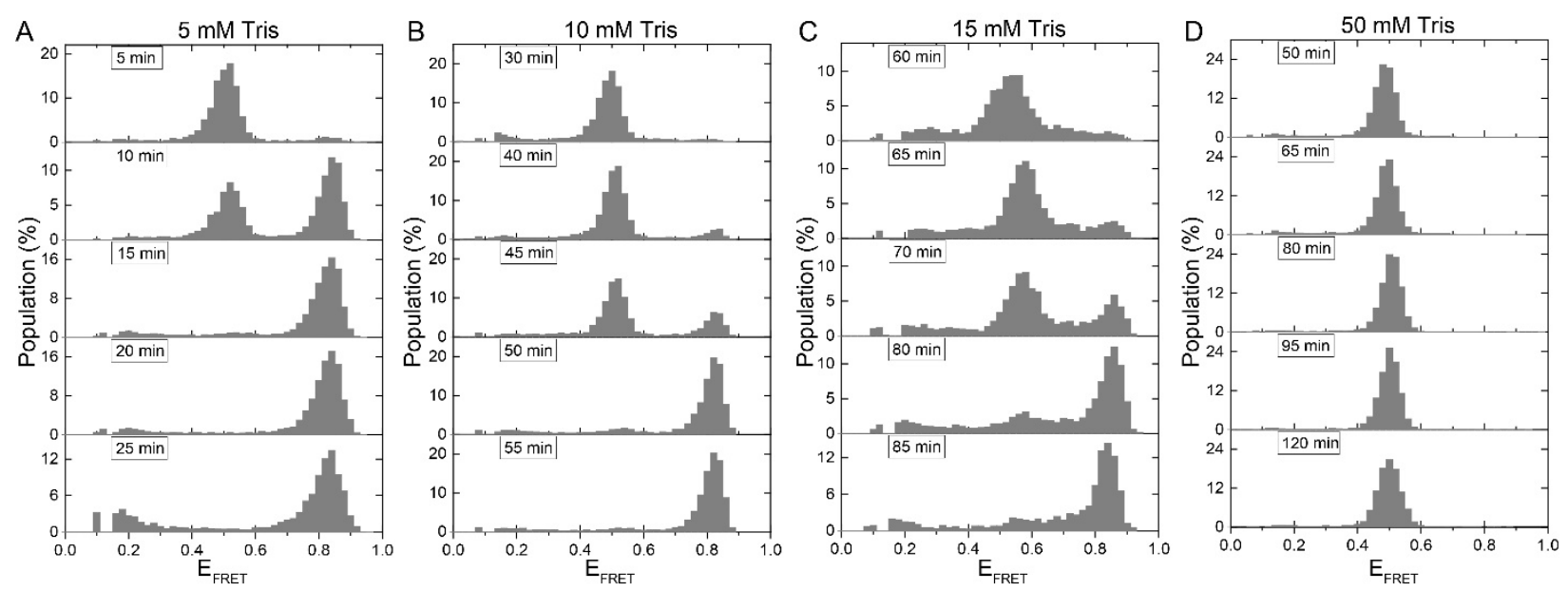

Figure 5 The use of $i$-motif as an in-situ $p H$ sensor for smFRET experiments. The gradual acidification of the environment when gloxy is used as the oxygen scavenging system is quantified for: (A) $5 \mathrm{mM}$ Tris; (B) $10 \mathrm{mM}$ Tris; (C) $15 \mathrm{mM}$ Tris; and (D) $50 \mathrm{mM}$ Tris. As the buffer strength is increased, the time it takes for the pH to drop from 7.5 to about 6.5, where i-motif folding starts, increases. At $50 \mathrm{mM}$ Tris, all the DNA molecules remain unfolded throughout the 120-minute imaging period. 
Figure 5 demonstrates these data where we periodically surveyed different regions of the chamber and quantified the fraction of molecules that remain unfolded or fold into i-motif after introducing gloxy into the chamber. These measurements show that all DNA molecules fold into i-motif after approximately $15 \mathrm{~min}$ in $5 \mathrm{mM}$ Tris, $55 \mathrm{~min}$ in $10 \mathrm{mM}$ Tris, and $85 \mathrm{~min}$ in $15 \mathrm{mM}$ Tris. These data set the time scale for $\mathrm{pH}$ to drop from 7.5 to around 6.5, where i-motif population becomes detectable, under these buffer conditions. The DNA molecules remain completely unfolded over 120 minutes of imaging in $50 \mathrm{mM}$ Tris. This suggests that the $\mathrm{pH}$ remains above 6.5 (based on Figure 2C-D) in $50 \mathrm{mM}$ Tris even after 120 minutes of incubating the gloxy in the chamber. These results are in quantitative agreement with the $\mathrm{pH}$ drop characterized by the ratiometric bulk FRET measurements on SNARF-1 ${ }^{58}$. It should be noted that the drop in the $\mathrm{pH}$ could be slowed down by using lower concentrations of glucose oxidase; however, this is often accompanied by lower photostability. In our experience, reducing the glucose oxidase concentration by a factor of 10 results in two-fold reduction in $\mathrm{Cy} 3 / \mathrm{Cy} 5$ photostability, while fluorophore brightness remains similar. For comparison, we also measured the stability of $\mathrm{pH}$ in PCA/PCD over a 120-min imaging period in $150 \mathrm{mM} \mathrm{KCl}$. Since we expect $\mathrm{pH}$ to be relatively stable in PCA/PCD, we selected a $\mathrm{pH}$ value where even small variations in $\mathrm{pH}$ would result in detectable changes in the fraction of the folded i-motif population. Based on the data in Figure $2 \mathrm{C}$ D, pH 6.3 would be the ideal choice for this purpose since approximately $50 \%$ of the molecules would be folded and small variations in the $\mathrm{pH}$ would result in significant changes in the folded fraction. We also used MES buffer for these studies since it is a better buffer in such lower $\mathrm{pH}$ studies. Figure 6 shows these data where the histograms after 30-min and 120-min are overlaid. The histograms are essentially identical, suggesting that the $\mathrm{pH}$ remains remarkably stable (6.29 \pm 0.05$)$ in PCA-PCD over 120-min imaging period. 


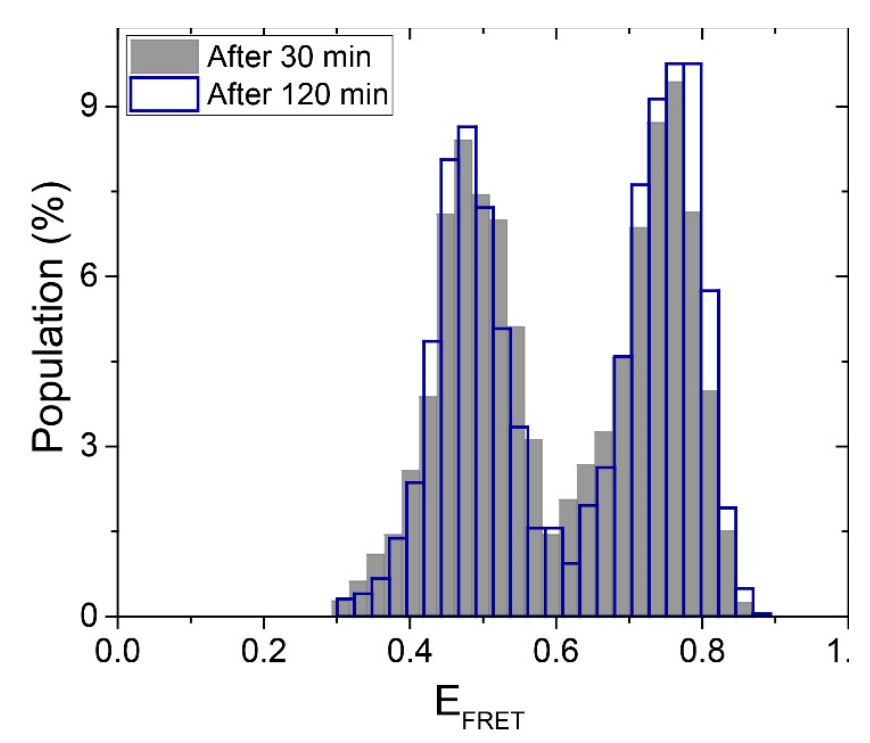

Figure 6 The stability of $\mathrm{pH}$ in PCA/PCD oxygen scavenging system. The FRET distribution essentially remains unchanged over 120-minute of imaging when PCA/PCD is used as the oxygen scavenging system, suggesting the pH remains constant to within \pm 0.05 of the initial pH of 6.3. The gray and blue histogram represent the distributions 30 min and 120 min, respectively, after incubation of $P C A / P C D$ in the chamber.

\section{Conclusion}

Our studies demonstrate that with proper design of the DNA construct and placement of the fluorophores, smFRET can be used to study i-motif systems over a broad range of ionic and $\mathrm{pH}$ conditions. Steady state distributions, dynamic transitions, and intermediate states during the folding process are clearly identifiable using different image acquisition approaches. The versatility of the method also suggests that interactions of i-motif structures with proteins, small molecules, or competing nucleic acid sequences would also be possible using smFRET. The imotif sequence we studied proved to be highly sensitive to $\mathrm{pH}$ variations in $6.3 \pm 0.3$ range. Based on this observation, we demonstrated the feasibility of using the i-motif as an in-situ $\mathrm{pH}$ sensor for SmFRET experiments to establish the time limits where the $\mathrm{pH}$ of the system drops below the physiologically relevant range.

Funding: National Institutes of Health [1R15GM109386 and 1R15GM123443 to H.B].

Conflict of interest statement. None declared. 


\section{References}

1. Gehring, K., Leroy, J. L. \& Guéron, M. A tetrameric DNA structure with protonated cytosine-cytosine base pairs. Nature 363, 561-565 (1993).

2. Phan, A. T., Guéron, M. \& Leroy, J.-L. The solution structure and internal motions of a fragment of the cytidine-rich strand of the human telomere. J. Mol. Biol. 299, 123-144 (2000).

3. Phan, A. T. \& Mergny, J. Human telomeric DNA: G-quadruplex, i-motif and Watson-Crick double helix. Nucleic Acids Res. 30, 4618-4625 (2002).

4. Parkinson, G. N., Lee, M. P. H. \& Neidle, S. Crystal structure of parallel quadruplexes from human telomeric DNA. Nature 417, 876-880 (2002).

5. Biver, T. Stabilisation of non-canonical structures of nucleic acids by metal ions and small molecules. Coord. Chem. Rev. 257, 2765-2783 (2013).

6. Wright, E. P., Huppert, J. L. \& Waller, Z. A. E. Identification of multiple genomic DNA sequences which form i-motif structures at neutral pH. Nucleic Acids Res. 45, 2951-2959 (2016).

7. McKim, M., Buxton, A., Johnson, C., Metz, A. \& Sheardy, R. D. Loop Sequence Context Influences the Formation and Stability of the i-Motif for DNA Oligomers of Sequence (CCCXXX)4, where X = A and/or T, under Slightly Acidic Conditions. J. Phys. Chem. B 120, 7652-7661 (2016).

8. Nguyen, T., Fraire, C. \& Sheardy, R. D. Linking pH, Temperature, and K+ Concentration for DNA i-Motif Formation. J. Phys. Chem. B 121, 7872-7877 (2017).

9. Liedl, T. \& Simmel, F. C. Switching the conformation of a DNA molecule with a chemical oscillator. Nano Lett. 5, 1894-1898 (2005).

10. Liu, D. \& Balasubramanian, S. A Proton-Fuelled DNA Nanomachine. Angew. Chemie - Int. Ed. 42, 5734-5736 (2003).

11. Shu, W. et al. DNA molecular motor driven micromechanical cantilever arrays. J. Am. Chem. Soc. 127, 17054-17060 (2005).

12. Gurung, S. P., Schwarz, C., Hall, J. P., Cardin, C. J. \& Brazier, J. A. The importance of loop length on the stability of i-motif structures. Chem. Commun. 51, 5630-5632 (2015).

13. Jin, K. S. et al. pH-dependent structures of an i-motif DNA in solution. J. Phys. Chem. B 113, 1852-1856 (2009). 
14. Mergny, J. L., Lacroix, L., Hélène, C., Han, X. \& Leroy, J. L. Intramolecular Folding of Pyrimidine Oligodeoxynucleotides into an i-DNA Motif. J. Am. Chem. Soc. 117, 88878898 (1995).

15. Kim, S. E., Lee, I. B., Hyeon, C. \& Hong, S. C. Destabilization of i-motif by submolar concentrations of a monovalent cation. J. Phys. Chem. B 118, 4753-4760 (2014).

16. Day, H. A., Wright, E. P., MacDonald, C. J., Gates, A. J. \& Waller, Z. A. E. Reversible DNA i-motif to hairpin switching induced by $\operatorname{copper}(<\mathrm{scp}>\mathrm{ii}</ \mathrm{scp}>$ ) cations. Chem. Commun. 51, 14099-14102 (2015).

17. Gao, J., Berden, G., Rodgers, M. T. \& Oomens, J. Interaction of $\mathrm{Cu}+$ with cytosine and formation of i-motif-like $\mathrm{C}-\mathrm{M}+-\mathrm{C}$ complexes: alkali versus coinage metals. Phys. Chem. Chem. Phys. 18, 7269-7277 (2016).

18. Cui, J., Waltman, P., Le, V. H. \& Lewis, E. A. The effect of molecular crowding on the stability of human c-MYC promoter sequence I-motif at neutral pH. Molecules 18, 1275112767 (2013).

19. Reilly, S. M., Morgan, R. K., Brooks, T. A. \& Wadkins, R. M. Effect of interior loop length on the thermal stability and p Kaof i-motif DNA. Biochemistry 54, 1364-1370 (2015).

20. Leroy, J. L., Gehring, K., Kettani, A. \& Guéron, M. Acid Multimers of Oligodeoxycytidine Strands: Stoichiometry, Base-Pair Characterization, and Proton Exchange Properties. Biochemistry 32, 6019-6031 (1993).

21. Ahmed, S., Kintanar, A. \& Henderson, E. Human telomeric c-strand tetraplexes. Nat. Struct. Biol. 1, 83-88 (1994).

22. Rajendran, A., Nakano, S. \& Sugimoto, N. Molecular crowding of the cosolutes induces an intramolecular i-motif structure of triplet repeat DNA oligomers at neutral $\mathrm{pH}$. Chem. Commun. 46, 1299 (2010).

23. Li, H., Hai, J., Zhou, J. \& Yuan, G. The formation and characteristics of the i-motif structure within the promoter of the c-myb proto-oncogene. J. Photochem. Photobiol. B Biol. 162, 625-632 (2016).

24. Sun, D. \& Hurley, L. H. The importance of negative superhelicity in inducing the formation of G-quadruplex and i-motif structures in the c-Myc promoter: Implications for drug targeting and control of gene expression. J. Med. Chem. 52, 2863-2874 (2009).

25. Day, H. A., Huguin, C. \& Waller, Z. A. E. Silver cations fold i-motif at neutral pH. Chem. 
Commun. 49, 7696 (2013).

26. Chen, Y. et al. Insights into the biomedical effects of carboxylated single-wall carbon nanotubes on telomerase and telomeres. Nat. Commun. 3, 1074 (2012).

27. Wang, Z. G., Elbaz, J. \& Willner, I. DNA machines: Bipedal walker and stepper. Nano Lett. 11, 304-309 (2011).

28. Liu, D. et al. A reversible pH-driven DNA nanoswitch array. J. Am. Chem. Soc. 128, 20672071 (2006).

29. Miyoshi, D., Inoue, M. \& Sugimoto, N. DNA logic gates based on structural polymorphism of telomere DNA molecules responding to chemical input signals. Angew. Chemie - Int. Ed. 45, 7716-7719 (2006).

30. Yang, Y. et al. An Electrochemically Actuated Reversible DNA Switch. Nano Lett. 10, 1393-1397 (2010).

31. Li, T., Ackermann, D., Hall, A. M. \& Famulok, M. Input-dependent induction of oligonucleotide structural motifs for performing molecular logic. J. Am. Chem. Soc. 134, 3508-3516 (2012).

32. Song, M. J. et al. Estimation of carrier frequencies of six autosomal-recessive Mendelian disorders in the Korean population. J. Hum. Genet. 57, 139-144 (2012).

33. Modi, S. et al. A DNA nanomachine that maps spatial and temporal $\mathrm{pH}$ changes inside living cells. Nat. Nanotechnol. 4, 325-330 (2009).

34. Surana, S., Bhat, J. M., Koushika, S. P. \& Krishnan, Y. An autonomous DNA nanomachine maps spatiotemporal $\mathrm{pH}$ changes in a multicellular living organism. Nat. Commun. 2, 340 (2011).

35. Zeraati, M. et al. I-motif DNA structures are formed in the nuclei of human cells. Nat. Chem. 10, 631-637 (2018).

36. Leroy, J. louis, Guéron, M., Mergny, J. louis \& Hélène, C. Intramolecular folding of a fragment of the cytosine-rich strand of telomeric DNA into an I-motif. Nucleic Acids Res. 22, 1600-1606 (1994).

37. Gallego, J., Chou, S. H. \& Reid, B. R. Centromeric pyrimidine strands fold into an intercalated motif by forming a double hairpin with a novel T:G:G:T tetrad: Solution structure of the d(TCCCGTTTCCA) dimer. J. Mol. Biol. 273, 840-856 (1997).

38. Khan, N. et al. Solution equilibria of the i-motif-forming region upstream of the B-cell 
lymphoma-2 P1 promoter. Biochimie 89, 1562-1572 (2007).

39. Bucek, P., Gargallo, R. \& Kudrev, A. Spectrometric study of the folding process of i-motifforming DNA sequences upstream of the c-kit transcription initiation site. Anal. Chim. Acta 683, 69-77 (2010).

40. Brazier, J. A., Shah, A. \& Brown, G. D. I-Motif formation in gene promoters: unusually stable formation in sequences complementary to known G-quadruplexes. Chem. Commun. 48, 10739 (2012).

41. Takahashi, S., Brazier, J. A. \& Sugimoto, N. Topological impact of noncanonical DNA structures on Klenow fragment of DNA polymerase. Proc. Natl. Acad. Sci. 114, 9605-9610 (2017).

42. Kang, H. J., Kendrick, S., Hecht, S. M. \& Hurley, L. H. The transcriptional complex between the BCL2 i-motif and hnRNP LL is a molecular switch for control of gene expression that can be modulated by small molecules. J. Am. Chem. Soc. 136, 4172-4185 (2014).

43. Kendrick, S. et al. The dynamic character of the BCL2 promoter i-motif provides a mechanism for modulation of gene expression by compounds that bind selectively to the alternative DNA hairpin structure. J. Am. Chem. Soc. 136, 4161-4171 (2014).

44. Sutherland, C., Cui, Y., Mao, H. \& Hurley, L. H. A Mechanosensor Mechanism Controls the G-Quadruplex/i-Motif Molecular Switch in the MYC Promoter NHE III1. J. Am. Chem. Soc. 138, 14138-14151 (2016).

45. Brown, R. V. et al. The Consequences of Overlapping G-Quadruplexes and i-Motifs in the Platelet-Derived Growth Factor Receptor $\beta$ Core Promoter Nuclease Hypersensitive Element Can Explain the Unexpected Effects of Mutations and Provide Opportunities for Selective Targeting of. J. Am. Chem. Soc. 139, 7456-7475 (2017).

46. Assi, H. A., Garavís, M., González, C. \& Damha, M. J. I-motif DNA: Structural features and significance to cell biology. Nucleic Acids Research 46, 8038-8056 (2018).

47. Selvam, S., Mandal, S. \& Mao, H. Quantification of Chemical and Mechanical Effects on the Formation of the G-Quadruplex and i-Motif in Duplex DNA. Biochemistry 56, 46164625 (2017).

48. Jonchhe, S. et al. Decreased water activity in nanoconfinement contributes to the folding of G-quadruplex and i-motif structures. Proc. Natl. Acad. Sci. U. S. A. 115, 9539-9544 (2018). 
49. Kim, S. E., Lee, I. B. \& Hong, S. C. The effect of the oxygen scavenging system on the pH of buffered sample solutions: In the context of single-molecule fluorescence measurements. Bull. Korean Chem. Soc. 33, 958-962 (2012).

50. Choi, J., Kim, S., Tachikawa, T., Fujitsuka, M. \& Majima, T. PH-induced intramolecular folding dynamics of i-motif DNA. J. Am. Chem. Soc. 133, 16146-16153 (2011).

51. Maleki, P. et al. Quantifying the impact of small molecule ligands on G-quadruplex stability against Bloom helicase. Nucleic Acids Res. (2019). doi:10.1093/nar/gkz803

52. Ray, S. et al. RPA-mediated unfolding of systematically varying G-quadruplex structures. Biophys. J. 104, 2235-2245 (2013).

53. Good, N. E. et al. Hydrogen Ion Buffers for Biological Research. Biochemistry 5, 467-477 (1966).

54. Adam, C., Olmos, J. M. \& Doneux, T. Electrochemical Monitoring of the Reversible Folding of Surface-Immobilized DNA i-Motifs. Langmuir 34, 3112-3118 (2018).

55. Tran, P. L., Mergny, J. L. \& Alberti, P. Stability of telomeric G-quadruplexes. Nucleic Acids Res 39, 3282-3294 (2011).

56. Bronson, J. E., Fei, J., Hofman, J. M., Gonzalez, R. L. \& Wiggins, C. H. Learning rates and states from biophysical time series: A Bayesian approach to model selection and singlemolecule FRET data. Biophys. J. 97, 3196-3205 (2009).

57. Lee, J. et al. Single-molecule four-color FRET. Angew. Chemie - Int. Ed. 49, 9922-9925 (2010).

58. Shi, X., Lim, J. \& Ha, T. Acidification of the oxygen scavenging system in single-molecule fluorescence studies: In situ sensing with a ratiometric dual-emission probe. Anal. Chem. 82, 6132-6138 (2010). 